December 2019 were invited to participate in the study in April 2021 by post. Under supervision of EORTC, the authors translated the disease specific EORTC QLQ-EN24 questionnaire to the Slovene language in accordance with EORTC guidelines. Demographic and clinical treatment data was evaluated and correlated with the QLQ-EN24 dimensions. Correlations were performed using the Spearman rank test, continuous data was compared using the Mann-Whitney U test. Data were evaluated using the SPSS for Mac version 23.0

Result(s)* Seventy-nine women participated in this study (response rate 51\%). Cronbach's alpha for items in the Slovenian version of the EORTC QLQ-EN24 scale was 0.72 . Median age of women was 64 years (36-85). Follow up time was 4 years (2-5). Sexual activity in the last 4 weeks prior to filling out the questionnaire was reported in 26 women (32\%). Median body mass index (BMI) was 31 (19-52). BMI was correlated with worse reported outcomes in lymphoedema $\left(\mathrm{r}_{\mathrm{s}}=-.246, \mathrm{p}<.045\right)$ and urological symptoms $\left(\mathrm{r}_{\mathrm{s}}=-.246\right.$, $\mathrm{p}<.044)$. Age was correlated only with items regarding poor body image $\left(r_{s}=-.350, p<.002\right)$, sexual interest $\left(r_{s}=-.408\right.$, $\mathrm{p}<.001)$ and sexual activity $\left(\mathrm{r}_{\mathrm{s}}=-.506, \mathrm{p}<.001\right)$. No other symptoms assessed were correlated with age. No patient recorded symptoms were correlated with surgery type (minimally invasive or open surgery) nor with lymph node treatment.

Conclusion* Our pilot study using a Slovenian version of the EORTC QLQ-EN24 showed adequate internal consistency. An initial analysis of the treatment mode did not impact patient reported health symptoms. There is a need for further understanding and support to women to prevent health symptoms post treatment and improve PROs.

\section{INDIVIDUALIZING SUPPORT TO IMPROVE QUALITY OF LIFE IN DIFFERENT PHASES OF BREAST CANCER TREATMENT}

M Rehrl ${ }^{*}$, LF Gantner, K Große Lackmann, J Ettl, M Kiechle, C Brambs. Technische Universität München, Frauenklinik rechts der Isar, Munich, Germany

\subsection{6/ijgc-2021-ESG0.568}

Introduction/Background* Due to the improved prognosis of patients with breast cancer, health-related quality of life has become increasingly important. The aim of the study is to evaluate the potential impact of different epidemiological, oncological and treatment parameters on the quality of life at different stages of diagnosis, treatment and follow-up in order to help improve and individualize the support for patients with breast cancer.

Methodology Between January 2019 and January 2021, 189 breast cancer patients were included. The quality of life was assessed using the European Organization for Research and Treatment of Cancer Quality of Life Questionnaire (EORTCQLQ-C30) and a German questionnaire quantifying psychological distress (FBK-R10) at three distinct time points: At initial diagnosis as well as after six and 12 months. Sociodemographic and clinical data were also included.

Result(s)* Both the subjective quality of life and the perceived health condition differed significantly between the three time points $(\mathrm{p}<0.01)$. After six months, the reported quality of life was significantly lower in all age groups. However, there was a significant improvement in the quality of life after 12 months. There was a trend to full rehabilitation in women age 50 to 69 . Further, quality of life was significantly lower in patients undergoing treatment compared to patients in follow-up $(p=0.01)$. Moreover, these patients suffered significantly more frequently from psychological distress $(p=0.035)$ and sexual dysfunction $(\mathrm{p}<0.05)$. There was a significant correlation between the EORTC and FBK-R10 questionnaires $(\mathrm{p}<0.05)$, suggesting a correlation between quality of life and psychological distress.

Conclusion* The quality of life decreased significantly during the first six months after diagnosis, identifying this time as a period of particular need for a multidisciplinary support system. In addition, patients undergoing treatment should receive special attention given their lower quality of life, greater psychological distress and substantially more sexual dysfunction. A significant improvement in quality of life can be observed 12 months after the initial diagnosis. Future studies should focus on how to regain an improved quality of life earlier and how to implement support systems based on the patients' different needs at different times during the course of the disease.

\section{ENHANCED RECOVERY AFTER SURGERY IS FEASIBLE, BENEFICIAL AND SHOULD BE THE STANDARD IN MAJOR GYNECOLOGICAL SURGERIES}

${ }^{1} \mathrm{~N}$ Kugelman*, ${ }^{1} \mathrm{O}$ Lavie, ${ }^{1} \mathrm{~N}$ Cohen, ${ }^{1} \mathrm{M}$ Schmidt, ${ }^{2} \mathrm{~A}$ Reuveni, ${ }^{1} \mathrm{~L}$ Ostrovsky, ${ }^{1} \mathrm{H}$ Dabah, ${ }^{1} Y$ Segev. ${ }^{1}$ Carmel Medical Center, Obstetrics and Gynecology, Rappaport Faculty of Medicine, Technion-Israel Institute of Technology, Haifa, Israel; ${ }^{2}$ Carmel Medical Center, Anesthesiology, Haifa, Israel

\subsection{6/ijgc-2021-ESG0.569}

Introduction/Background* Enhanced recovery after surgery (ERAS) protocols are evidence-based protocols designed to standardize medical care, improve outcomes, and lower health care costs. Our objective was to evaluate the implementation of the ERAS protocol, and its effect on recovery during the hospitalization period after gynecological laparotomy surgeries.

Methodology In this retrospective cohort study we compared demographic and clinical data of consecutive patients at a single institute who underwent open gynecological surgeries before (August 2017- December 2018) and after (January 2019- March 2020) the implementation of the ERAS protocol. Eighty women were included in each group.

Result(s)* The clinical and demographic characteristics were similar between the women operated before and after implementation of the ERAS protocol. Following implementation of the protocol, decreases were observed in post-surgical hospitalization (from $4.89 \pm 2.56$ to $4.09 \pm 1.65$ days; $p=0.01$ ), in patients reporting nausea symptoms (from $18(22.5 \%)$ to 7 $(8.8 \%) ; \mathrm{p}=0.017)$, and in the use of postoperative opioids (from $77(96.3 \%)$ to $47(58.8 \%) ; \mathrm{p}<0.001)$. No significant changes were identified between the two periods regarding vomiting, 30-day re-hospitalization and postoperative minor and major complications.

Conclusion* Implementation of the ERAS protocol is feasible and was found to result in less postoperative opioid use, a faster return to normal feeding and a shorter postoperative hospital stay. Implementation of the protocol implementation was not associated with an increased rate of complications nor with re-admissions. 